\title{
Blood lactate dynamics in awake and anaesthetized mice after intraperitoneal and subcutaneous injections of lactate - sex matters
}

\author{
Øyvind P. Haugen ${ }^{1}$, Evan M. Vallenari ${ }^{1}$, Imen Belhaj ${ }^{1,2}$, Milada C. Småstuen ${ }^{3}$, Jon Storm-Mathisen ${ }^{2}$, Linda H. \\ Bergersen $^{\text {Corresp., } 1}$, Ingrid Åmellem ${ }^{\text {Corresp. } 1}$ \\ ${ }^{1}$ The Brain and Muscle Energy Group, Electron Microscopy Laboratory, Institute of Oral Biology, University of Oslo, Oslo, Norway \\ 2 Amino Acid Transporter Laboratory, Division of Anatomy, Department of Molecular Medicine, Institute of Basic Medical Sciences, SERTA: Healthy Brain \\ Ageing Centre, University of Oslo, Oslo, Norway \\ 3 Department of Nursing and Health Promotion, Faculty of Health Science, Oslo Metropolitan University, Oslo, Norway \\ Corresponding Authors: Linda H. Bergersen, Ingrid Åmellem \\ Email address: I.h.bergersen@odont.uio.no, ingrid.amellem@odont.uio.no
}

Lactate treatment has shown a therapeutic potential for several neurological diseases, including Alzheimer's disease. In order to optimize the administration of lactate for studies in mouse models, we compared blood lactate dynamics after intraperitoneal (IP) and subcutaneous (SC) injections. We used the 5xFAD mouse model for familial Alzheimer's disease and performed the experiments in both awake and anaesthetized mice. Blood glucose was used as an indication of the hepatic conversion of lactate. In awake mice, both injection routes resulted in high blood lactate levels, mimicking levels reached during highintensity training. In anaesthetized mice, SC injections resulted in significantly lower lactate levels compared to IP injections. Interestingly, we observed that awake males had significantly higher lactate levels than awake females, while the opposite sex difference was observed during anaesthesia. We did not find any significant difference between transgenic and wild-type mice and therefore believe that our results can be generalized to other mouse models. These results should be considered when planning experiments using lactate treatment in mice. 
1 BLOOD LACTATE DYNAMICS IN AWAKE AND ANAESTHETIZED MICE AFTER

2 INTRAPERITONEAL AND SUBCUTANEOUS INJECTIONS OF LACTATE - SEX MATTERS

3

4 Øyvind P. Haugen ${ }^{1}$, Evan M. Vallenari ${ }^{1}$, Imen Belhaj ${ }^{1,2}$, Milada C. Småstuen ${ }^{3}$, Jon Storm-

5 Mathisen $^{2}$, Linda H. Bergersen ${ }^{1^{*}}$, Ingrid Åmellem ${ }^{1^{*}}$

6

7 1. The Brain and Muscle Energy Group, Electron Microscopy Laboratory, Institute of Oral

8 Biology, University of Oslo, Norway.

9 2. Amino Acid Transporter Laboratory, Division of Anatomy, Department of Molecular

10 Medicine, Institute of Basic Medical Sciences, SERTA: Healthy Brain Ageing Centre, University

11 of Oslo, Norway.

12 3. Department of Nursing and Health Promotion, Faculty of Health Science, Oslo Metropolitan

13 University, Oslo, Norway.

14

15

* Corresponding authors: Linda H. Bergersen: 1.h.bergersen@odont.uio.no, Ingrid Åmellem: ingrid.amellem@odont.uio.no

\section{ABSTRACT}

Lactate treatment has shown a therapeutic potential for several neurological diseases, including Alzheimer's disease. In order to optimize the administration of lactate for studies in mouse models, we compared blood lactate dynamics after intraperitoneal (IP) and subcutaneous (SC) injections. We used the $5 \mathrm{xFAD}$ mouse model for familial Alzheimer's disease and performed the experiments in both awake and anaesthetized mice. Blood glucose was used as an indication of the hepatic conversion of lactate. In awake mice, both injection routes resulted in high blood lactate levels, mimicking levels reached during high-intensity training. In anaesthetized mice, SC injections resulted in significantly lower lactate levels compared to IP injections. significant difference between transgenic and wild-type mice and therefore believe that our results can be generalized to other mouse models. These results should be considered when planning experiments using lactate treatment in mice. 


\section{INTRODUCTION}

33 Physical exercise has beneficial effects on health, both in preventing and treating several diseases. Among these are neurological and psychiatric diseases, and physical exercise may delay the progression of Alzheimer's disease (Tari et al. 2019). Blood lactate levels can increase several-fold during high-intensity training (Goodwin et al. 2007). Some of the lactate produced during exercise crosses the blood-brain barrier and enters the brain, where it can be metabolised (Pierre and Pellerin 2005; van Hall et al. 2009). In men performing high-intensity interval training, improvement in executive functions correlated with blood lactate levels and cerebral lactate uptake (Hashimoto et al. 2018). Systemic lactate administration has been shown to increase levels of brain-derived neurotrophic factor (BDNF) in both humans and mice at rest and improved learning and memory in mice (El Hayek et al. 2019; Schiffer et al. 2011). BDNF facilitates neuronal growth, differentiation, survival, and synaptic plasticity, and plays a vital role in the pathophysiology of several neurological disorders (Mitre, Mariga, and Chao 2017).

We have previously identified the $\mathrm{G}_{\mathrm{i}}$-coupled lactate receptor HCAR 1 in the brain. Notably, lactate injections mimicked the effect of high-intensity interval training by increasing the combined neuro-angiotrophic factor VEGF and angiogenesis in the hippocampus of wild-type mice, but not in HCAR1 knockout mice (Morland et al. 2017). This effect of HCAR1 activation can potentially delay the progression of vascular dementias and Alzheimer's disease. In addition, lactate has been shown to protect against inflammation through an HCAR1-mediated mechanism (Hoque et al. 2014), which may be an important component of the pathology of Alzheimer's disease (Kinney et al. 2018). Thus, based on the present knowledge, lactate administration has therapeutic potential for several neurological diseases.

Intraperitoneal (IP) and subcutaneous (SC) injections are common routes for drug delivery in rodents. Even though both injection types are considered parenteral, differences in absorption rate and bioavailability can be expected. Drugs administered through SC injection are absorbed through capillaries in the subcutis and often slower than other parenteral administration routes, which in turn can result in a more sustained effect (Turner et al. 2011). IP injection resembles to some extent an oral administration route, where drugs are absorbed from the gastrointestinal tract into the portal vein for delivery to the liver. Drugs injected into the peritoneal cavity are absorbed mainly through the mesenteric vessels that drain into the portal vein. Through monocarboxylate transporters, lactate enters hepatic cells, where it can be metabolised. The lactate can be converted to pyruvate in the liver and used as substrate for the synthesis of 
66 glucose through gluconeogenesis (Brooks 1986). Some of the lactate injected IP will therefore

67 undergo hepatic metabolism before reaching the systemic blood flow. Similarly, some of the

68 lactate injected SC or produced and released by skeletal muscles during anaerobic exercise, may

69 eventually be metabolised in the liver.

In order to optimize the administration of lactate for studies of the mouse brain, we compared blood lactate dynamics after IP and SC injections in a mouse model (5xFAD) of Alzheimer's disease and in corresponding wild-type control mice (Oakley et al. 2006). Blood glucose was measured to monitor the conversion of lactate to glucose by the liver. Comparison of these injection methods for lactate has to our knowledge not been performed in any mouse model. Injections were performed both on awake and anaesthetized mice, induced by isoflurane, which is the most common volatile anaesthetic agent used in laboratory mice (Gargiulo et al. 2012). These two conditions might result in different response curves.

\section{MATERIALS AND METHODS}

2.1 Animals

The mice used in this study (5xFAD) (Oakley et al. 2006) were obtained from the Mutant Mouse Resource \& Research Centers (MMRRC strain name B6SJLTg(APPSwFlLon,PSEN1*M146L*L286V)6799Vas/Mmjax; stock number 034840-JAX). They were bred and treated in accordance with the national and regional ethical guidelines. All experiments were performed by certified personnel and approved by the Animal Use and Care Committee of the Institute of Basic Medical Sciences, The Faculty of Medicine, University of Oslo, and by the Norwegian Animal Research Authority (FOTS 17448, 17551, 15525). All the mice were housed under a 12:12 hour light/dark cycle and had free access to water and chow.

\subsection{Group distributions}

92 Genotypes (wild-type and transgenic) were verified by conventional PCR and gel

93 electrophoresis. The mice were randomly divided into different groups based on treatment, with

94 5-6 mice in each group. Sex and genotype distributions in the different groups did not exceed

$952 / 3$ in favour of one or another, and the average age was between 12 and 17 weeks. See

96 Supplementary Table 1 for a complete overview of the different groups.

97

$98 \quad 2.3$ Lactate injections 
99 Mice subjected to lactate injections received sodium L-lactate (71718, Sigma-Aldrich, MO, USA);

$1002 \mathrm{~g} / \mathrm{kg}$ body weight; $200 \mathrm{mg} / \mathrm{ml}$ dissolved in PBS, $\mathrm{pH} 7.4$; corresponding to $18 \mathrm{mmol} / \mathrm{kg}$, by IP

101 injection or SC injection on the neck/back (Fig. 1A and B). Control mice got the same volume of

102 PBS injected (per kg bodyweight). The concentration of sodium L-lactate mimics lactate levels

103 reached after high-intensity training (Morland et al. 2017).

104

105

2.4 Anaesthesia

106

Mice subjected to anaesthesia were exposed to isoflurane in an airflow chamber with 400 $\mathrm{ml} / \mathrm{min}$ airflow with $4 \%$ isoflurane prior to blood samplings and injections (Baxter, San Juan, Puerto Rico). Anaesthesia was maintained with $200 \mathrm{ml} / \mathrm{min}$ airflow with 1.7-2.5\% isoflurane. A heating block was placed beneath the airflow chamber to maintain a stable body temperature.

2.5 Blood samplings and measurements

112 Blood samples were taken immediately before (baseline) and at the following time points after

113 injection: 5, 13, 37 minutes for the awake mice. The time course was extended to include 60

114 minutes for mice under anaesthesia to account for potentially delayed absorption and clearance

115 rates. By puncturing the submandibular vein with a $5 \mathrm{~mm}$ long Goldenrod animal lancet

116 (Braintree Scientific, Inc, MA, USA), $20 \mu$ of blood was collected with a capillary tube (Fig. 1C)

117 and immediately emptied into a pre-filled Eppendorf tube with haemolysing solution (EKF

118 Diagnostics, Cardiff, UK) with subsequent shaking. The samples were analysed for lactate and

119 glucose levels on a Biosen C-Line GP+ system (EKF Diagnostics, Cardiff, UK). Due to practical

120 reasons making it challenging to collect blood samples at the exact same time points for all

121 animals, we calculated the average time points and used linear interpolation to obtain lactate

122 and glucose levels (Fig. 2). The range and average of the actual times are shown in

123 Supplementary Table 2.

\subsection{Statistical analysis}

126 Possible differences between groups were analysed using a linear mixed effect regression model

127 for repeated measurements. The analyses were performed separately for awake and

128 anaesthetized mice. The model was fitted separately for the two outcomes, lactate and glucose,

129 and the covariates age, sex and genotype were included in the model. In addition, an interaction

130 term "time* group" was fitted to estimate possible differences in time trajectories between the

131 groups. The results are presented as the estimated means with standard error of the regression 
132 (S.E.R.). To correct for multiple testing, significance level was set to 0.01. All analyses were 133 performed using Stata ver. 14.2 (StataCorp LLC, TX, USA).

134

\section{RESULTS AND DISCUSSION}

136 The baseline levels of blood lactate (group averages ranging from about 6.5 to 9.2 $\mathrm{mM}$ in awake

137 mice, Fig. 2A) were higher than expected. Normal physiological blood lactate in mice has

138 previously been reported to be in the range of 2.5 to $4.6 \mathrm{mM}$ (Iversen et al. 2012; Schwarzkopf et

139 al. 2013). A possible explanation to the high baseline is that the injections, and especially the

140 blood sampling, could be experienced as stressful, causing the lactate levels to rise. This

141 interpretation is supported by the fact that the blood glucose levels continued to rise during the

142 experimental period in the awake mice (Fig. 2C). Both hyperlactataemia and hyperglycaemia are

143 well-known adrenergic responses (Gjedsted et al. 2011; Levy et al. 2003). In fact, repeated blood

144 sampling with two minute intervals has been shown to increase plasma glucose in a time-

145 dependent manner in mice (Tabata, Kitamura, and Nagamatsu 1998). A stress-induced

146 adrenergic response is expected to be diminished by anaesthesia, but we did not see a change in

147 the baseline lactate levels after isoflurane exposure (Fig. 2B). It has been proposed that

148 isoflurane and other volatile anaesthetics can alter mitochondrial function and thereby affect the

149 synthesis and/or degradation of lactate (Horn and Klein 2010).

150

151 In anaesthetized mice, lactate injected IP gave significantly higher lactate levels (24.9 mM, 95\%

152 CI [20.6 to 29.2]) than SC injected (15.3 mM, 95\% CI [11.4 to 19.2]) after 13 minutes (Fig. 2B).

153 Although we did not find statistically significant differences at other time points in the

154 anaesthetized mice nor in the awake mice at any time point, there is a trend showing higher

155 lactate levels with IP injection than SC injection. This is consistent with the observation that SC

156 injections have the slowest absorption rate of parenteral administration routes (Turner et al.

157 2011).

158

159 Since lactate injected IP enters the liver through the portal vein, it was of interest to examine

160 whether lactate was lost by hepatic conversion to glucose. Administration of lactate in rats has

161 been shown to induce hypophagia when injected IP, while no effect on food intake was found in

162 rats receiving SC injections (Racotta and Russek 1977). The authors speculated that this is due to

163 a hepatic action, which probably is mediated by hepatic glucoreceptors. However, we did not

164 find any statistically significant changes in blood glucose between the groups, although the

165 glucose values were somewhat higher after IP than after SC injections at later time points during 
166 anaesthesia (Fig. 2D). We cannot exclude the possibility that some of the lactate was used as a

167 gluconeogenic precursor without affecting blood glucose, which has been shown with ${ }^{13} \mathrm{C}$

168 labelled lactate infusions in humans (Miller et al. 2002). Despite this, the high blood lactate

169 levels observed after injections suggest that most of the IP injected lactate reached the systemic

170 blood.

171

172 Previous studies have shown that isoflurane induces hyperglycaemia by inhibiting insulin

173 secretion (Schwarzkopf et al. 2013; Zuurbier et al. 2014; Windeløv, Pedersen, and Holst 2016).

174 Our data are consistent with the values reported by Schwarzkopf et al. (Schwarzkopf et al. 2013).

175 In general, different anaesthetics are known to affect metabolism and various physiological

176 characteristics, and it is important to be aware of such confounding effects. In studies that aim

177 to look at the effects of a specific metabolite, the use of anaesthesia should be carefully

178 considered. We used isoflurane in this study, as it is the most common anaesthetic used in mice.

179 Compared to the results obtained from awake mice (Fig. 2A), blood lactate levels after injections

180 during anaesthesia changed at a slower rate, with the highest levels being lower and observed at

181 later time points (Fig. 2C). This agrees with observations of protracted tissue distribution time

182 courses of various markers in isoflurane anaesthesia compared to the awake state (Avram et al.

183 2000). As the lactate levels seemed to be sustained for a longer time during anaesthesia, we

184 included measurements at 60 minutes. The levels were approximately twice as high as baseline

185 after 60 minutes during anaesthesia, while they were closer to baseline after 37 minutes in the

186 awake mice. The protracted time course of blood lactate after SC compared to IP injection in

187 anaesthetized mice agrees with the observation that blood flow in skin and fat is about one

188 seventh of that in the small intestine of mice anaesthetized with isoflurane (Boswell et al. 2014).

190 While neither age nor genotype showed statistically significant effects on blood glucose or

191 lactate levels, sex had a significant effect on the lactate levels both during awake and

192 anaesthetized conditions. When awake, male mice had higher lactate levels than female mice by

193 an overall mean difference of $3.3 \mathrm{mM}$ (95\% CI [1.1 to 5.46], p < 0.01). During isoflurane-induced

194 anaesthesia, lactate levels in male mice were more than halved, dropping to a lower level than in

195 female mice by an overall mean difference of $3.0 \mathrm{mM}$ (95\% CI [1.87 to 4.1], p < 0.001). Lactate

196 levels in female mice did not seem to be notably affected by the isoflurane. The sex differences

197 estimated by the statistical analysis can clearly be seen when presenting the time course for each

198 sex separately, regardless of injection method (Fig. 3). 
200 Sex differences in blood lactate levels have, as far as we know, not been reported in mice, and we

201 can only hypothesize on these findings. A possibility is that, when awake, male mice respond

202 differently to handling than females due to a more aggressive nature. However, in a study by

203 Hurst and West, differences in stress and anxiety responses after routine laboratory handling

204 showed only minor sex differences (Hurst and West 2010). Differences in muscle physiology

205 could also influence lactate levels, as males generally have more glycolytic muscle fibres than

206 females (Haizlip, Harrison, and Leinwand 2015). Since adrenaline can induce hyperlactataemia

207 (Gjedsted et al. 2011; Levy et al. 2003), it is possible that different muscle fibres could produce

208 different amounts of lactate in response to the stress of handling and blood sampling. Whatever

209 the reason, the male-to-female difference in $\mathrm{mM}$ lactate was present before lactate injections

210 and also occurred after PBS injections in awake mice (Fig. 3A). Different lactate levels in males

211 and females were also observed during anaesthesia, which indicates a sex dependent effect of

212 the anaesthetic. A study performed on rats showed that brief exposure to isoflurane elevates

213 plasma corticosteroid levels in females, but not in males (Bekhbat et al. 2016). Such an effect

214 might explain why females have higher lactate levels than males after exposure to isoflurane

215 (Fig. 3B), opposite to what is observed in awake mice. It is possible that elevated stress

216 hormones after exposure to isoflurane prevent lactate levels in females from declining. Future

217 studies including measurements of stress markers are needed to elucidate these sex differences.

218

219 4. CONCLUSION

220 Based on our findings, both IP and SC injections are suitable options for administration of

221 lactate in mice. In spite of some differences in the dynamics, both injection routes yielded high

222 blood lactate levels, mimicking levels reached during high-intensity training. When performing

223 lactate injections under isoflurane-induced anaesthesia, one should be aware that SC injections

224 could result in significantly lower lactate levels than IP injections. There were sex-dependent

225 differences in lactate levels in both awake and anaesthetized conditions. The dynamics, as well

226 as the possible sex-differences, should be taken into account when using mouse models for

227 studies involving lactate treatments.

228

229

230

231

REFERENCES

232

Peer) reviewing PDF | (2019:10:41879:1:1:NEW 27 Nov 2019) 
233

234

235

236

237

238

239

240

241

242

243

244

245

246

247

248

249

250

251

252

253

254

255

256

257

258

259

260

261

262

263

264

265

266

267

268

269

270

271

272

273

274

275

276

277

278

279

280

281

282

Avram, M. J., T. C. Krejcie, C. U. Niemann, C. Enders-Klein, C. A. Shanks, and T. K. Henthorn. 2000. 'Isoflurane alters the recirculatory pharmacokinetics of physiologic markers', Anesthesiology, 92: 1757-68.

Bekhbat, Mandakh, Liana Merrill, Sean D. Kelly, Vanessa K. Lee, and Gretchen N. Neigh. 2016. 'Brief anesthesia by isoflurane alters plasma corticosterone levels distinctly in male and female rats: Implications for tissue collection methods', Behavioural brain research, 305: 122-25.

Boswell, C. A., E. E. Mundo, S. Ulufatu, D. Bumbaca, H. S. Cahaya, N. Majidy, M. Van Hoy, M. G. Schweiger, P. J. Fielder, S. Prabhu, and L. A. Khawli. 2014. 'Comparative physiology of mice and rats: radiometric measurement of vascular parameters in rodent tissues', Mol Pharm, 11: 1591-8.

Brooks, G. A. 1986. 'The lactate shuttle during exercise and recovery', Med Sci Sports Exerc, 18: 360-8.

El Hayek, L., M. Khalifeh, V. Zibara, R. Abi Assaad, N. Emmanuel, N. Karnib, R. El-Ghandour, P. Nasrallah, M. Bilen, P. Ibrahim, J. Younes, E. Abou Haidar, N. Barmo, V. Jabre, J. S. Stephan, and S. F. Sleiman. 2019. 'Lactate Mediates the Effects of Exercise on Learning and Memory through SIRT1-Dependent Activation of Hippocampal Brain-Derived Neurotrophic Factor (BDNF)', J Neurosci, 39: 2369-82.

Gargiulo, S., A. Greco, M. Gramanzini, S. Esposito, A. Affuso, A. Brunetti, and G. Vesce. 2012. 'Mice anesthesia, analgesia, and care, Part I: anesthetic considerations in preclinical research', ILAR J, 53: E55-69.

Gjedsted, J., M. Buhl, S. Nielsen, O. Schmitz, E. T. Vestergaard, E. Tonnesen, and N. Moller. 2011. 'Effects of adrenaline on lactate, glucose, lipid and protein metabolism in the placebo controlled bilaterally perfused human leg', Acta Physiol (Oxf), 202: 641-8.

Goodwin, Matthew L., James E. Harris, Andrés Hernández, and L. Bruce Gladden. 2007. 'Blood lactate measurements and analysis during exercise: a guide for clinicians', Journal of diabetes science and technology, 1: 558-69.

Haizlip, K. M., B. C. Harrison, and L. A. Leinwand. 2015. 'Sex-based differences in skeletal muscle kinetics and fiber-type composition', Physiology (Bethesda, Md.), 30: 30-39.

Hashimoto, T., H. Tsukamoto, S. Takenaka, N. D. Olesen, L. G. Petersen, H. Sorensen, H. B. Nielsen, N. H. Secher, and S. Ogoh. 2018. 'Maintained exercise-enhanced brain executive function related to cerebral lactate metabolism in men', Faseb j, 32: 1417-27.

Hoque, R., A. Farooq, A. Ghani, F. Gorelick, and W. Z. Mehal. 2014. 'Lactate reduces liver and pancreatic injury in Toll-like receptor- and inflammasome-mediated inflammation via GPR81-mediated suppression of innate immunity', Gastroenterology, 146: 1763-74.

Horn, Tobias, and Jochen Klein. 2010. 'Lactate levels in the brain are elevated upon exposure to volatile anesthetics: A microdialysis study', Neurochem Int, 57: 940-47.

Hurst, J. L., and R. S. West. 2010. 'Taming anxiety in laboratory mice', Nat Methods, 7: 825-6.

Iversen, Nina K., Hans Malte, Erik Baatrup, and Tobias Wang. 2012. 'The normal acid-base status of mice', Respiratory Physiology \& Neurobiology, 180: 252-57.

Kinney, Jefferson W., Shane M. Bemiller, Andrew S. Murtishaw, Amanda M. Leisgang, Arnold M. Salazar, and Bruce T. Lamb. 2018. 'Inflammation as a central mechanism in Alzheimer's disease', Alzheimer's \& dementia (New York, N. Y.), 4: 575-90.

Levy, Bruno, Arnaud Mansart, Pierre-Edouard Bollaert, Patricia Franck, and Jean-Pierre Mallie. 2003. 'Effects of epinephrine and norepinephrine on hemodynamics, oxidative metabolism, and organ energetics in endotoxemic rats', Intensive Care Medicine, 29: 292-300.

Miller, B. F., J. A. Fattor, K. A. Jacobs, M. A. Horning, F. Navazio, M. I. Lindinger, and G. A. Brooks. 2002. 'Lactate and glucose interactions during rest and exercise in men: effect of exogenous lactate infusion', $J$ Physiol, 544: 963-75.

Peer] reviewing PDF | (2019:10:41879:1:1:NEW 27 Nov 2019) 
283

284

285

286

287

288

289

290

291

292

293

294

295

296

297

298

299

300

301

302

303

304

305

306

307

308

309

310

311

312

313

314

315

316

317

318

319

320

321

322

323

324

325

326

Mitre, M., A. Mariga, and M. V. Chao. 2017. 'Neurotrophin signalling: novel insights into mechanisms and pathophysiology', Clin Sci (Lond), 131: 13-23.

Morland, C., K. A. Andersson, O. P. Haugen, A. Hadzic, L. Kleppa, A. Gille, J. E. Rinholm, V. Palibrk, E. H. Diget, L. H. Kennedy, T. Stolen, E. Hennestad, O. Moldestad, Y. Cai, M. Puchades, S. Offermanns, K. Vervaeke, M. Bjoras, U. Wisloff, J. Storm-Mathisen, and L. H. Bergersen. 2017. 'Exercise induces cerebral VEGF and angiogenesis via the lactate receptor HCAR1', Nat Commun, 8: 15557.

Oakley, H., S. L. Cole, S. Logan, E. Maus, P. Shao, J. Craft, A. Guillozet-Bongaarts, M. Ohno, J. Disterhoft, L. Van Eldik, R. Berry, and R. Vassar. 2006. 'Intraneuronal beta-amyloid aggregates, neurodegeneration, and neuron loss in transgenic mice with five familial Alzheimer's disease mutations: potential factors in amyloid plaque formation', $J$ Neurosci, 26: 10129-40.

Pierre, K., and L. Pellerin. 2005. 'Monocarboxylate transporters in the central nervous system: distribution, regulation and function', $J$ Neurochem, 94: 1-14.

Racotta, R., and M. Russek. 1977. 'Food and water intake of rats after intraperitoneal and subcutaneous administration of glucose, glycerol and sodium lactate', Physiol Behav, 18: 267-73.

Schiffer, T., S. Schulte, B. Sperlich, S. Achtzehn, H. Fricke, and H. K. Struder. 2011. 'Lactate infusion at rest increases BDNF blood concentration in humans', Neurosci Lett, 488: 234-7.

Schwarzkopf, T. M., T. Horn, D. Lang, and J. Klein. 2013. 'Blood gases and energy metabolites in mouse blood before and after cerebral ischemia: the effects of anesthetics', Exp Biol Med (Maywood), 238: 84-9.

Tabata, H., T. Kitamura, and N. Nagamatsu. 1998. 'Comparison of effects of restraint, cage transportation, anaesthesia and repeated bleeding on plasma glucose levels between mice and rats', Lab Anim, 32: 143-8.

Tari, Atefe R., Cecilie S. Norevik, Nathan R. Scrimgeour, Asgeir Kobro-Flatmoen, Jon StormMathisen, Linda H. Bergersen, Christiane D. Wrann, Geir Selbæk, Miia Kivipelto, José Bianco N. Moreira, and Ulrik Wisløff. 2019. 'Are the neuroprotective effects of exercise training systemically mediated?', Progress in Cardiovascular Diseases.

Turner, Patricia V., Thea Brabb, Cynthia Pekow, and Mary Ann Vasbinder. 2011. 'Administration of substances to laboratory animals: routes of administration and factors to consider', Journal of the American Association for Laboratory Animal Science : JAALAS, 50: 600-13.

van Hall, G., M. Stromstad, P. Rasmussen, O. Jans, M. Zaar, C. Gam, B. Quistorff, N. H. Secher, and H. B. Nielsen. 2009. 'Blood lactate is an important energy source for the human brain', J Cereb Blood Flow Metab, 29: 1121-9.

Windeløv, Johanne A., Jens Pedersen, and Jens J. Holst. 2016. 'Use of anesthesia dramatically alters the oral glucose tolerance and insulin secretion in $\mathrm{C} 57 \mathrm{Bl} / 6$ mice', Physiol Rep, 4: e12824.

Zuurbier, C. J., A. Koeman, S. M. Houten, M. W. Hollmann, and W. J. Florijn. 2014. 'Optimizing anesthetic regimen for surgery in mice through minimization of hemodynamic, metabolic, and inflammatory perturbations', Exp Biol Med (Maywood), 239: 737-46.

Peer) reviewing PDF | (2019:10:41879:1:1:NEW 27 Nov 2019) 
Figure 1

Sites of injection and blood sampling.

Intraperitoneal (IP) (A) and subcutaneous (SC) (B) injections in mice. Blood samples were collected from the submandibular vein (C). Illustrated with BioRender.

A

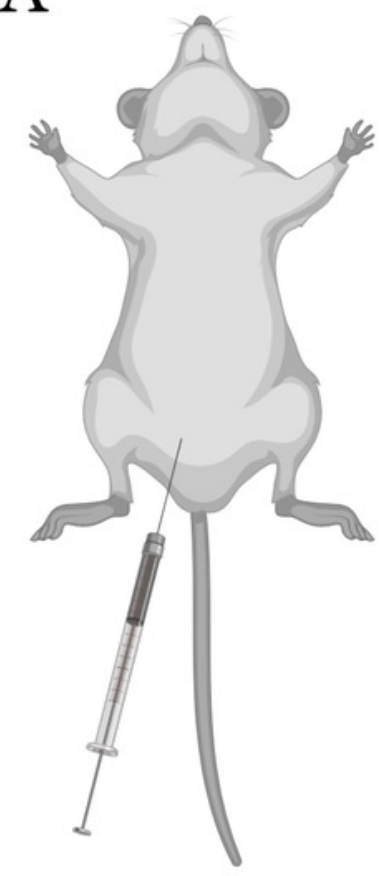

B

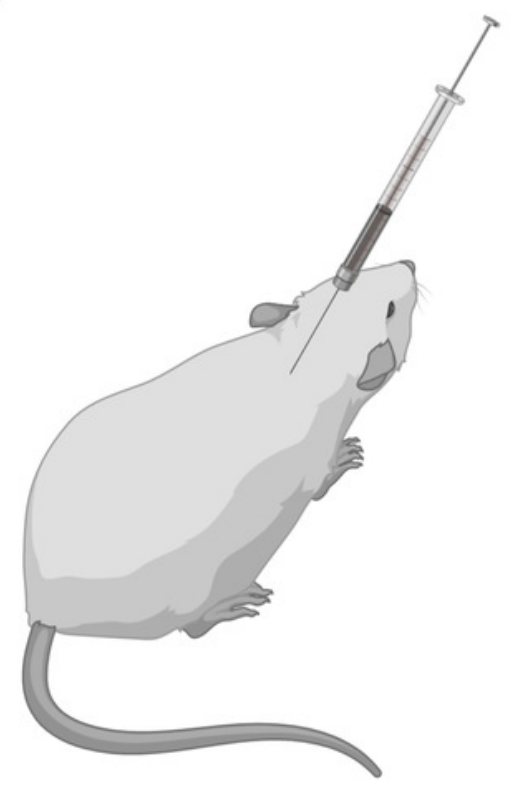

C

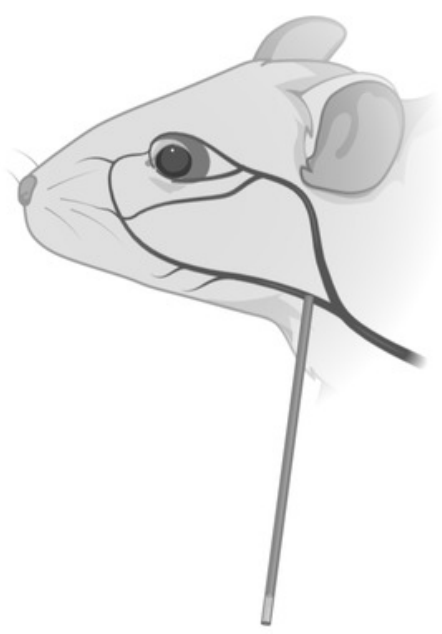


Figure 2

Time-response for blood lactate and glucose.

Lactate (A, B) and glucose (C, D) measured in blood samples taken from the submandibular vein in awake $(A, C)$ or anaesthetized mice $(B, D)$. Lactate or PBS were injected intraperitoneally (IP) or subcutaneously (SC). Blood samples were taken immediately before (0) and at 5, 13 and $37 \mathrm{~min}$ after injection for the awake mice and extended to $60 \mathrm{~min}$ for the anaesthetized mice. The data are presented as the estimated mean \pm S.E.R., * equals $p<$ 0.01 . Statistical analyses were done using a linear mixed effect regression model for repeated measurements.
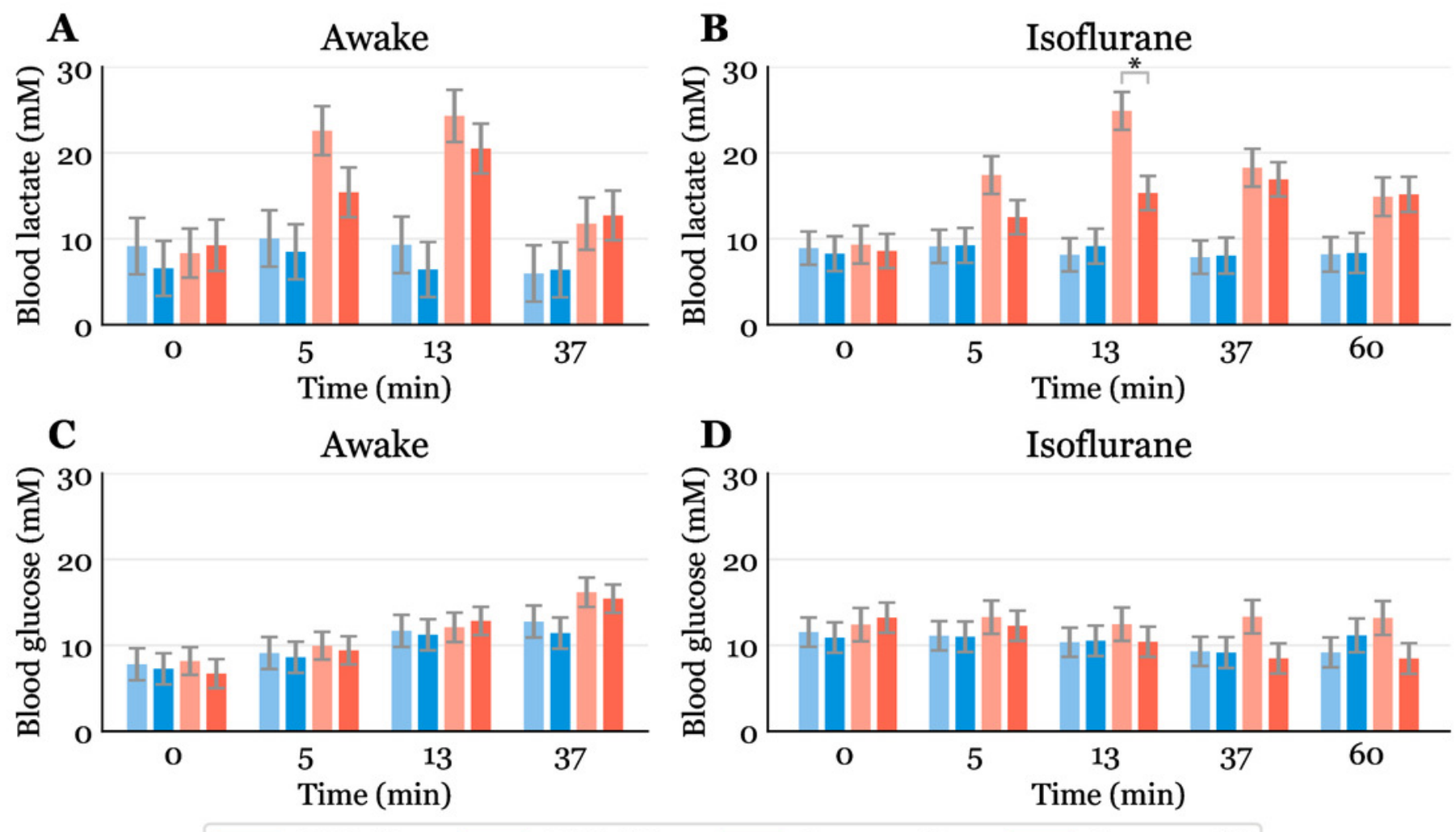

PBS, IP PBS, SC

Lactate, IP

Lactate, SC 


\section{Figure 3}

Time-response for blood lactate and glucose by sex.

Mice that received intraperitoneal (IP) or subcutaneous (SC) injection were pooled, giving one lactate injected group and one PBS injected group for each sex (awake lactate groups:

females $n=7$, males $n=5$; isoflurane lactate groups: females $n=6$, males $n=6$; awake PBS groups: females $n=5$, males $n=6$; isoflurane PBS groups: females $n=5$, males $n=6$ ). Lactate $(A, B)$ and glucose $(C, D)$ measured in blood samples taken from the submandibular vein in awake $(A, C)$ or anaesthetized mice $(B, D)$. Blood samples were taken immediately before (0) and at 5, 13 and $37 \mathrm{~min}$ after injection for the awake mice and extended to $60 \mathrm{~min}$ for the anaesthetized mice. The data are presented as the estimated mean \pm S.E.R.
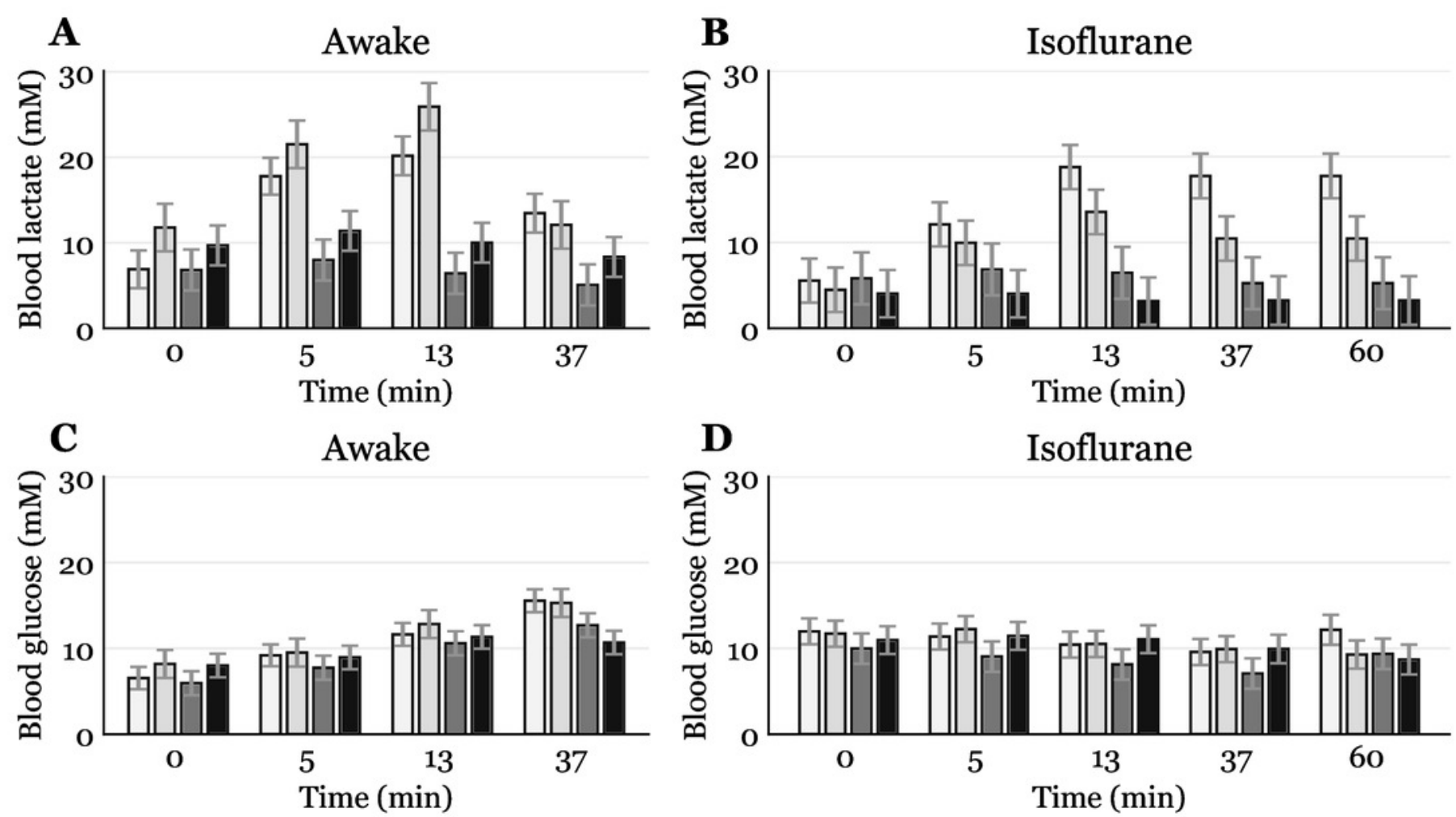

Female, lactate

Male, lactate

Female, PBS

Male, PBS 